\title{
Mathematical morphology operators over concept lattices
}

\author{
Jamal Atif ${ }^{1}$, Isabelle Bloch ${ }^{2}$, Felix Distel $^{3}$, and Céline Hudelot ${ }^{4}$ \\ 1 Université Paris Sud, LRI - TAO, Orsay, France. \\ 2 Institut Mines Telecom - Telecom ParisTech - CNRS LTCI, Paris, France. \\ 3 Technische Universität Dresden - Fakultät Informatik - Institut für theoretische \\ Informatik, Dresden, Germany. \\ 4 MAS Laboratory, Ecole Centrale de Paris, France.
}

\begin{abstract}
Although mathematical morphology and formal concept analysis are two lattice-based data analysis theories, they are still developed in two disconnected research communities. The aim of this paper is to contribute to fill this gap, beyond the classical relationship between the Galois connections defined by the derivation operators and the adjunctions underlying the algebraic mathematical morphology framework. In particular we define mathematical morphology operators over concept lattices, based on distances, valuations, or neighborhood relations in concept lattices. Their properties are also discussed. These operators provide new tools for reasoning over concept lattices.
\end{abstract}

\section{Introduction}

Formal concept analysis and mathematical morphology are two important theories used for knowledge representation and information processing, and are both based on lattice theory. Although they have been mostly developed independently, their common algebraic framework leads to similarities that deserve to be investigated. In this paper, we propose to contribute to filling this gap by establishing some links between both domains. Several applications can benefit from such links. For instance, non-monotonic reasoning operators can be proposed [1], by exploiting also the links between formal concept analysis and description logics [2] (in earlier works morphological reasoning operators have been proven to be interesting in the case of propositional logics, e.g. [7]). Besides, these operators can be exploited in the context of logical concept analysis [8] to propose new reasoning services. Extensions to fuzzy formal concept analysis, based on fuzzy mathematical morphology [6] can then be derived (with links also with rough sets and fuzzy rough sets [4]).

Our main contributions are definitions of mathematical morphology operators on concept lattices, which provide tools for handling information represented by pairs of objects and attributes.

In Section 2, we recall some basic definitions and notations of both domains, which will be used in the following. In Section 3, we highlight some similarities 
between two basic morphological operators, dilation and erosion, and the derivation operators commonly used in formal concept analysis. Our main contribution is then developed in Section 4, where we propose original definitions of dilations and erosions, on the power set of objects (or attributes), and on the concept lattice itself. The proposed definitions are based on distances, valuations, and neighborhood relations. They enjoy good formal properties.

\section{Preliminaries}

\subsection{Formal Concept Analysis}

We assume the reader to be familiar with formal concept analysis (FCA) [9], and restrict this section to the introduction of notations, and of some definitions and results that will be useful in the sequel. A formal context is defined as a triple $\mathbb{K}=(G, M, I)$, where $G$ is the set of objects, $M$ the set of attributes, and $I \subseteq G \times M$ a relation between the objects and the attributes. A pair $(g, m) \in I$ stands for "the object $g$ has the attribute $m$ ". The formal concepts of the context $\mathbb{K}$ are all pairs $(X, Y)$ with $X \subseteq G$ and $Y \subseteq M$ such that $(X, Y)$ is maximal with the property $X \times Y \subseteq I$. The set $X$ is called the extent and the set $Y$ is called the intent of the formal concept $(X, Y)$. For any formal concept $a$, we denote its extent by $e(a)$ and its intent by $i(a)$, i.e. $a=(e(a), i(a))$. The set of all formal concepts of a given context can be hierarchically ordered by inclusion of their extent: $\left(X_{1}, Y_{1}\right) \preceq\left(X_{2}, Y_{2}\right) \Leftrightarrow X_{1} \subseteq X_{2}$ ( $\left.\Leftrightarrow Y_{2} \subseteq Y_{1}\right)$. This order, that reflects the subconcept-superconcept relation, always induces a complete lattice which is called the concept lattice of the context $(G, M, I)$, denoted $\mathbb{C}(\mathbb{K})$. For $X \subseteq G$ and $Y \subseteq M$, the derivation operators $\alpha$ and $\beta$ are defined as $\alpha(X)=\{m \in M \mid \forall g \in$ $X,(g, m) \in I\}$, and $\beta(Y)=\{g \in G \mid \forall m \in Y,(g, m) \in I\}$.

Definition 1. Let $(L, \preceq)$ and $\left(L^{\prime}, \preceq^{\prime}\right)$ be two partially ordered sets. A pair of operators $(\alpha, \beta), \alpha: L \rightarrow L^{\prime}, \beta: L^{\prime} \rightarrow L$, defines a Galois-connection if $\forall x \in$ $L, \forall y \in L^{\prime}, y \preceq{ }^{\prime} \alpha(x) \Longleftrightarrow x \preceq \beta(y)$.

The pair $(\alpha, \beta)$ is a Galois connection between the partially ordered power sets $(\mathcal{P}(G), \subseteq)$ and $(\mathcal{P}(M), \subseteq)$. Therefore, for $X_{1} \subseteq X_{2} \subseteq G$ (resp. $\left.Y_{1} \subseteq Y_{2} \subseteq M\right)$, the following holds: (i) $\alpha\left(X_{2}\right) \subseteq \alpha\left(X_{1}\right)$ (resp. $\beta\left(Y_{2}\right) \subseteq \beta\left(Y_{1}\right)$ ), (ii) $X_{1} \subseteq \beta\left(\alpha\left(X_{1}\right)\right.$ ) and $\alpha\left(X_{1}\right)=\alpha\left(\beta\left(\alpha\left(X_{1}\right)\right)\right)$ (resp. $Y_{1} \subseteq \alpha\left(\beta\left(Y_{1}\right)\right)$ and $\beta\left(Y_{1}\right)=\beta\left(\alpha\left(\beta\left(Y_{1}\right)\right)\right)$ ). Saying that $(X, Y)$ with $X \subseteq G$ and $Y \subseteq M$ is a formal concept is equivalent to $\alpha(X)=Y$ and $\beta(Y)=X$. Given an element $a \in \mathbb{C}$, the set $F_{a}=\{b \in \mathbb{C} \mid a \preceq b\}$ is called the principal filter generated by the element $a$. The set $I_{a}=\{b \in \mathbb{C} \mid$ $b \preceq a\}$ is called the principal ideal generated by the concept $a$.

Theorem 1 ([9]). In a concept lattice, infimum and supremum of a family of formal concepts $\left(X_{t}, Y_{t}\right)_{t \in T}$ are given by:

$$
\begin{aligned}
& \bigwedge_{t \in T}\left(X_{t}, Y_{t}\right)=\left(\bigcap_{t \in T} X_{t}, \alpha\left(\beta\left(\bigcup_{t \in T} Y_{t}\right)\right)\right), \\
& \bigvee_{t \in T}\left(X_{t}, Y_{t}\right)=\left(\beta\left(\alpha\left(\bigcup_{t \in T} X_{t}\right)\right), \bigcap_{t \in T} Y_{t}\right)
\end{aligned}
$$


Every complete lattice can be viewed as a concept lattice. A complete lattice $(L, \preceq)$ is isomorphic to the concept lattice $\mathbb{C}(L, L, \preceq)$.

In Section 4 we introduce morphological operators defined on the powerset of the set of objects or the set of attributes. Two mappings will be of particular interest: attribute concept and object concept.

Definition 2 (Object and attribute concept). Let $(G, M, I)$ be a context with the associated concept lattice $\mathbb{C}$. Let $g \in G$ be an object and $m \in M$ an attribute. The concept $\gamma g=(\beta \alpha(g), \alpha(g))$ is called the object concept of $g$ and the concept $\mu m=(\beta(m), \alpha \beta(m))$ is called the attribute concept of $m$. We define the mappings $p_{\mathbb{C}}$ and $q_{\mathbb{C}}$ which project objects and attributes to their respective object and attribute concepts as follows.

$$
p_{\mathbb{C}}:\left|\begin{array}{l}
G \longrightarrow \mathbb{C} \\
g \longmapsto \gamma g
\end{array} \quad q_{\mathbb{C}}:\right| \begin{aligned}
& M \longrightarrow \mathbb{C} \\
& m \longmapsto \mu m
\end{aligned}
$$

b

Theorem 2 ([9]). Let $(G, M, I)$ be a context with associated (complete) concept lattice $\mathbb{C}$ and let $p_{\mathbb{C}}$ and $q_{\mathbb{C}}$ be mappings as defined in Definition 2. Then, $p_{\mathbb{C}}(G)$ is join-dense in $\mathbb{C}$ and $q_{\mathbb{C}}(M)$ is meet-dense in $\mathbb{C}$. Also $(g, m) \in I$ is equivalent to $p_{\mathbb{C}}(g) \preceq q_{\mathbb{C}}(m)$ for all $g \in G$ and all $m \in M$, with the usual partial ordering on $\mathbb{C}$.

Every concept $(X, Y)$ is the join of all its sub-object-concepts, and is the meet of its super-attribute-concepts:

$$
(X, Y)=\bigvee_{g \in X} p_{\mathbb{C}}(g)=\bigwedge_{m \in Y} q_{\mathbb{C}}(m) .
$$

Let us now introduce other decompositions of concepts. Note that decompositions of a concept as a supremum (respectively as an infimum) will be directly used to compute dilations (respectively erosions) in Section 4.3.

Definition 3. An element $a$ in the lattice $\mathbb{C}$ is join-irreducible if (i) $a \neq 0_{\mathbb{C}}$ (where $0_{\mathbb{C}}$ denotes the least element of the lattice), and (ii) $\forall(a, b) \in \mathbb{C}^{2}, a=$ $b \vee c \Rightarrow a=b$ or $a=c$. The set of all join-irreducible elements in $\mathbb{C}$ is denoted by $\mathcal{J}(\mathbb{C})$ and the set of all meet-irreducible elements (defined in a similar way) by $\mathcal{M}(\mathbb{C})$.

Every element in $\mathbb{C}$ can be written as the supremum of a set of join-irreducible elements in $\mathbb{C}$ and as the infimum of a set of meet-irreducible elements in $\mathbb{C}$ :

$$
\forall a \in \mathbb{C}, a=\bigvee\{b \in \mathcal{J}(\mathbb{C}) \mid b \preceq a\}=\bigwedge\{b \in \mathcal{M}(\mathbb{C}) \mid a \preceq b\}
$$

Note that join-irreducible and meet-irreducible decompositions of an element $a$ in $\mathbb{C}$ are not unique in general unless the lattice enjoys some local distributivity properties [20]. We consider here a minimal decomposition that is intended in the following sense: 
Definition 4. Let $A, B \subseteq \mathbb{C}$, we say that $A$ refines $B$ (written $A \ll B$ ) if for each $a \in A$ there exists $b \in B$ with $a \preceq b$. A set $B \subseteq \mathcal{J}(\mathbb{C})$ is called a joinirreducible decomposition of $a$ in $\mathbb{C}$ iff $\bigvee B=a . B$ is said to be minimal if every join-irreducible decomposition $C$ of a satisfies $C \ll B \Longrightarrow B \subseteq C$. (Minimal) meet-irreducible decompositions can be defined analogously.

Example 1. We consider a classical example to illustrate the definitions introduced above. Furthermore this example will be used throughout the paper to illustrate and discuss the proposed operators. The considered formal context and the associated concept lattice are depicted in Figure 1.

\begin{tabular}{|l||c|c|c|c|c|}
\hline $\mathbb{K}$ & composite & even & odd & prime & square \\
\hline \hline 1 & & & $\times$ & & $\times$ \\
\hline 2 & & $\times$ & & $\times$ & \\
\hline 3 & & & $\times$ & $\times$ & \\
\hline 4 & $\times$ & $\times$ & & & $\times$ \\
\hline 5 & & & $\times$ & $\times$ & \\
\hline 6 & $\times$ & $\times$ & & & \\
\hline 7 & & & $\times$ & $\times$ & \\
\hline 8 & $\times$ & $\times$ & & & \\
\hline 9 & $\times$ & & $\times$ & & $\times$ \\
\hline 10 & $\times$ & $\times$ & & & \\
\hline
\end{tabular}

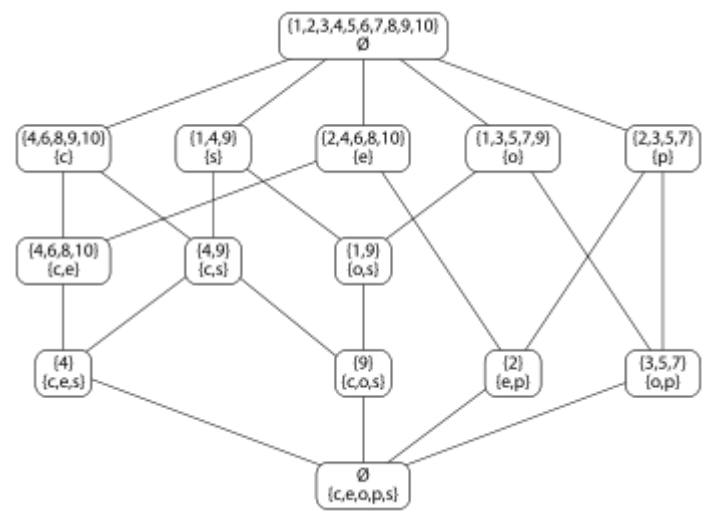

Fig. 1. A simple example of a context and its concept lattice from Wikipedia (objects are integers from 1 to 10, and attributes are composite, even, odd, prime and square).

\subsection{Mathematical Morphology}

Mathematical morphology [17], in its deterministic part dealing with increasing operators, usually relies on the algebraic framework of complete lattices [3]. Let us first recall this basic algebraic framework. Let $(L, \preceq)$ and $\left(L^{\prime}, \preceq^{\prime}\right)$ be two complete lattices (which do not need to be equal). All the following definitions and results are common to the general algebraic framework of mathematical morphology in complete lattices $[10,11,15,18]$.

Different terminologies can be found in different lattice theory related contexts (refer to [16] for equivalence tables). Some of these equivalences will be given in Section 3.

Definition 5. An operator $\delta: L \rightarrow L^{\prime}$ is a dilation if it commutes with the supremum (sup-preserving mapping): $\forall\left(x_{i}\right) \in L, \delta\left(\vee_{i} x_{i}\right)=\vee_{i}^{\prime} \delta\left(x_{i}\right)$, where $\vee$ denotes the supremum associated with $\preceq$ and $\vee^{\prime}$ the one associated with $\preceq^{\prime}$. 
An operator $\varepsilon: L^{\prime} \rightarrow L$ is an erosion if it commutes with the infimum (infpreserving mapping): $\forall\left(x_{i}\right) \in L^{\prime}, \varepsilon\left(\wedge_{i}^{\prime} x_{i}\right)=\wedge_{i} \varepsilon\left(x_{i}\right)$, where $\wedge$ and $\wedge^{\prime}$ denote the infimum associated with $\preceq$ and $\preceq^{\prime}$, respectively.

Algebraic dilations $\delta$ and erosions $\varepsilon$ are increasing operators; moreover $\delta$ preserves the smallest element and $\varepsilon$ preserves the largest element.

A fundamental notion in this algebraic framework is the one of adjunction.

Definition 6. A pair of operators $(\varepsilon, \delta), \delta: L \rightarrow L^{\prime}, \varepsilon: L^{\prime} \rightarrow L$, defines an adjunction if $\forall x \in L, \forall y \in L^{\prime}, \delta(x) \preceq^{\prime} y \Longleftrightarrow x \preceq \varepsilon(y)$.

The main properties, that will be used in the following, are summarized as follows.

Proposition $1([\mathbf{1 0}, \mathbf{1 1}, \mathbf{1 5}, \mathbf{1 8}])$. If a pair of operators $(\varepsilon, \delta)$ defines an adjunction, then the following results hold:

$-\delta$ preserves the smallest element and $\varepsilon$ preserves the largest element;

$-\delta$ is a dilation and $\varepsilon$ is an erosion (in the sense of Definition 5);

- $\delta \varepsilon$ is anti-extensive: $\delta \varepsilon \preceq^{\prime} I d_{L^{\prime}}$, where $I d_{L^{\prime}}$ denotes the identity mapping on $L^{\prime}$, and $\varepsilon \delta$ is extensive: $I d_{L} \preceq \varepsilon \delta$. The compositions $\delta \varepsilon$ and $\varepsilon \delta$ are called morphological opening and morphological closing, respectively;

- $\varepsilon \delta \varepsilon=\varepsilon, \delta \varepsilon \delta=\delta, \delta \varepsilon \delta \varepsilon=\delta \varepsilon$ and $\varepsilon \delta \varepsilon \delta=\varepsilon \delta$, i.e. morphological opening and closing are idempotent operators.

Let $\delta$ and $\varepsilon$ be two increasing operators such that $\delta \varepsilon$ is anti-extensive and $\varepsilon \delta$ is extensive. Then $(\varepsilon, \delta)$ is an adjunction.

The following representation result also holds. If $\varepsilon$ is an increasing operator, it is an algebraic erosion if and only if there exists $\delta$ such that $(\varepsilon, \delta)$ is an adjunction. The operator $\delta$ is then an algebraic dilation and can be expressed as $\delta(x)=\bigwedge^{\prime}\left\{y \in L^{\prime} \mid x \preceq \varepsilon(y)\right\}$. A similar representation result holds for erosion.

Particular forms of dilations and erosions can be defined based on the notion of a structuring element, which can be a neighborhood relation or any binary relation. Let us for instance assume that $L$ and $L^{\prime}$ are power sets of some sets, denoted by $L=\mathcal{P}(M)$ and $L^{\prime}=\mathcal{P}(G)$, and let $I$ be a binary relation between $G$ and $M$. By construction $\left(L, L^{\prime}, I\right)$ is a formal context if $G, M$ and $I$ are defined as in Section 2.1.

A structuring element centered at $m \in M$, or a neighborhood of $m$, is the set of $g \in G$ such that $(g, m) \in I$. Morphological dilations and erosions are then defined as:

$$
\begin{array}{ll}
\forall Y \in \mathcal{P}(M), & \delta_{I}(Y)=\{g \in G \mid \exists m \in Y,(g, m) \in I\} \\
\forall X \in \mathcal{P}(G), & \varepsilon_{I}(X)=\{m \in M \mid \forall g \in G,(g, m) \in I \Longrightarrow g \in X\} .
\end{array}
$$

Using the FCA derivation operators on the context $(G, M, I)$ the operators $\delta_{I}$ and $\varepsilon_{I}$ can be expressed as: $\delta_{I}(Y)=\bigcup_{m \in Y} \beta(m)$, and $\varepsilon_{I}(X)=\{m \mid \alpha(m) \subseteq X\}$.

If $L=L^{\prime}$, we have the following equivalences: 
$I$ reflexive $\Longleftrightarrow \delta_{I}$ extensive $\Longleftrightarrow \varepsilon_{I}$ anti-extensive.

Other properties can be found e.g. in [5].

As an example, let us consider that $L=L^{\prime}=\mathcal{P}\left(\mathbb{R}^{2}\right)$, with set inclusion as partial ordering. Let us denote by $B$ a structuring element (subset of $\mathbb{R}^{2}$ ), $\check{B}$ its symmetrical with respect to the origin of the space, and $B_{x}$ its translation at point $x$. Translation invariant operators are then expressed as: $\forall X \subseteq \mathbb{R}^{2}$, $\delta_{B}(X)=\left\{x \in \mathbb{R}^{2} \mid \check{B}_{x} \cap X \neq \emptyset\right\}, \varepsilon_{B}(X)=\left\{x \in \mathbb{R}^{2} \mid B_{x} \subseteq X\right\}$. These operations are illustrated in Figure 2 for $B$ being a ball of the Euclidean distance. Intuitively, the dilation extends $X$ by an amount corresponding to the radius of the structuring element, while erosion reduces $X$. Dilation may connect connected components and close holes, while erosion may suppress some connected components, or parts of them that are smaller than the structuring element (in terms of inclusion).

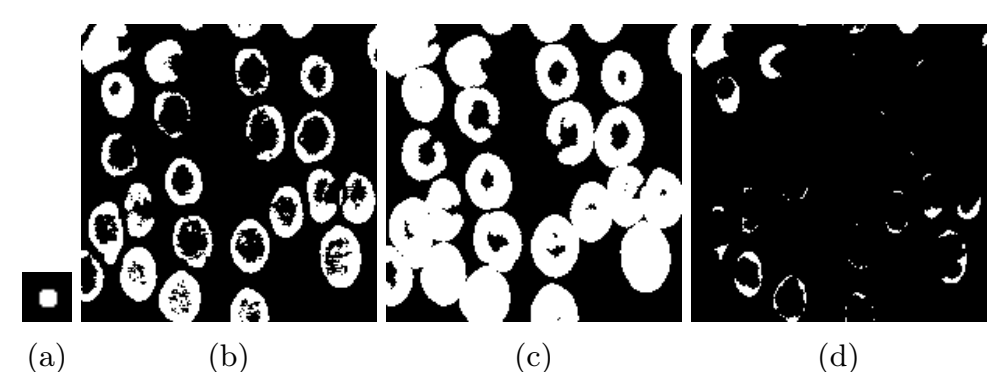

Fig. 2. (a) Structuring element $B$ (ball of the Euclidean distance). (b) Subset $X$ in the Euclidean plane (in white). (c) Its dilation $\delta_{B}(X)$. (d) Its erosion $\varepsilon_{B}(X)$.

\section{A First Link between FCA and Mathematical Morphology}

As already briefly noticed e.g. in [5] FCA and mathematical morphology both rely on complete lattice structures which share some similarities. In this section, we highlight some parallel properties of dilations and erosions on the one hand, and of derivation operators on the other hand. The first important link is that $(\varepsilon, \delta)$ is an adjunction (sometimes called monotone Galois connection), while $(\alpha, \beta)$ is an antitone Galois connection. It is obvious from Definitions 1 and 6 that the two properties are equivalent if we reverse the order for one of the lattices. The same holds for all properties derived from adjunctions or Galois connections (cf. Proposition 1). The most important ones are summarized in Table $1^{5}$.

One should note that the two fields typically use different terminology for equivalent concepts: increasing, idempotent and extensive operator are called

\footnotetext{
${ }^{5}$ In the table we denote by $\operatorname{Inv}(\varphi)$ the set of invariants of an operator $\varphi$ (i.e. $x \in \operatorname{Inv}(\varphi)$ iff $\varphi(x)=x)$.
} 
Table 1. Similarities between Mathematical Morphology and Formal Concept Analysis.

\begin{tabular}{ll}
\hline Adjunctions, dilations and erosions & Galois connection, derivation operators \\
\hline$\delta: L \rightarrow L^{\prime}, \varepsilon: L^{\prime} \rightarrow L$ & $\alpha: \mathcal{P}(G) \rightarrow \mathcal{P}(M), \beta: \mathcal{P}(M) \rightarrow \mathcal{P}(G)$ \\
$\delta(x) \preceq \preceq^{\prime} y \Longleftrightarrow x \preceq \varepsilon(y)$ & $X \subseteq \alpha(Y) \Longleftrightarrow Y \subseteq \beta(X)$ \\
increasing operators & decreasing operators \\
$\varepsilon \delta \varepsilon=\varepsilon, \delta \varepsilon \delta=\delta$ & $\alpha \beta \alpha=\alpha, \beta \alpha \beta=\beta$ \\
$\varepsilon \delta$ closing (closure operator), $\delta \varepsilon$ opening & $\alpha \beta$ and $\beta \alpha$ both closure operators (closings) \\
(kernel operator) & \\
$\operatorname{Inv}(\varepsilon \delta)=\varepsilon\left(L^{\prime}\right), \operatorname{Inv}(\delta \varepsilon)=\delta(L)$ & $\operatorname{Inv}(\alpha \beta)=\alpha(\mathcal{P}(G)), \operatorname{Inv}(\beta \alpha)=\beta(\mathcal{P}(M))$ \\
$\varepsilon\left(L^{\prime}\right)$ is a Moore family, & $\alpha(\mathcal{P}(G))$ and $\beta(\mathcal{P}(M))$ are Moore families \\
$\delta(L)$ is a dual Moore family & $($ or closure systems) \\
$\delta$ is a dilation: $\delta\left(\vee x_{i}\right)=\vee^{\prime}\left(\delta\left(x_{i}\right)\right)$ & $\alpha$ is an anti-dilation: $\alpha\left(\cup Y_{i}\right)=\cap \alpha\left(Y_{i}\right)$ \\
$\varepsilon$ is an erosion: $\varepsilon\left(\wedge^{\prime} y_{i}\right)=\wedge\left(\varepsilon\left(y_{i}\right)\right)$ & $\beta$ is an anti-dilation: $\beta\left(\cup X_{i}\right)=\cap \beta\left(X_{i}\right)$ \\
\hline
\end{tabular}

closings in MM and closure operators in FCA, while increasing, idempotent and anti-extensive operators are called openings in MM and kernel operators in FCA. Similarly, in FCA literature it is more common to speak of closure systems, instead of Moore families.

In the following sections, we go beyond this simple translation of terminology from one theory to the other. We propose new morphological operators acting on concept lattices. These operators can then be used to reason on such lattices.

\section{Mathematical Morphology Operators over Concept Lattices}

Here we consider operators over the concept lattice $(\mathbb{C}, \preceq)$, denoted as $\mathbb{C}$ in the sequel when no confusion occurs, associated with a given context $\mathbb{K}=(G, M, I)$. As in any complete lattice, we define dilations and erosions in the concept lattice as operations that commute with the supremum and the infimum, respectively.

In the following, we propose two approaches to concretely define these operations on $(\mathbb{C}, \preceq$ ): The first one (Section 4.2 ) is based on the notion of structuring element, defined as an elementary neighborhood of elements of $G$ or as a binary relation between elements of $G$. We define such a neighborhood as a ball of radius 1 of some distance function on $G$ derived from a distance on $\mathbb{C}$. The second approach (Section 4.3) defines morphological operators directly from a distance on $\mathbb{C}$.

While defining morphological operators on the power set of a given set by using a structuring element is common in the mathematical morphology community, defining these operators directly from a distance on the underlying lattice $(\mathbb{C}$ here) is completely new. 
Since the notion of distance is central for both approaches, we discuss in the next subsection how to construct metrics on concept lattices, based in particular on valuations.

\subsection{Distances from Valuations on $(\mathbb{C}, \preceq)$}

Let us first introduce a lattice metric from any function defined on $\mathbb{C}$.

Definition 7. Let $f$ be a real-valued decreasing function on $\mathbb{C}$. If a and $b$ are elements of $\mathbb{C}$ such that $b$ covers $a$, then the lattice metric $d$ is defined as

$$
d(a, b)=d(b, a)=f(b)-f(a)
$$

For any other elements, we define

$$
d(a, b)=\inf \sum_{i=1}^{n} d\left(a_{i}, a_{i-1}\right)
$$

where $a=a_{0}, a_{1}, \cdots, a_{n}=b$ is a path from a to $b$ (i.e. either $a_{i-1}$ covers $a_{i}$ or the contrary), and the infimum is taken over all paths.

With additional properties on the real-valued function, some metrics or pseudo-metrics (i.e. the separability axiom may not hold) can be defined explicitly, without requiring the computation of the infimum over a set of paths.

Definition 8. Let $(\mathbb{C}, \preceq)$ be a concept lattice. A real-valued function $\omega$ on $(\mathbb{C}, \preceq)$ is a lower valuation if it satisfies the following (supermodular) property:

$$
\forall\left(a_{1}, a_{2}\right) \in \mathbb{C}^{2}, \omega\left(a_{1}\right)+\omega\left(a_{2}\right) \leq \omega\left(a_{1} \wedge a_{2}\right)+\omega\left(a_{1} \vee a_{2}\right),
$$

and is an upper valuation if it satisfies the following (submodular) property:

$$
\forall\left(a_{1}, a_{2}\right) \in \mathbb{C}^{2}, \omega\left(a_{1}\right)+\omega\left(a_{2}\right) \geq \omega\left(a_{1} \wedge a_{2}\right)+\omega\left(a_{1} \vee a_{2}\right)
$$

A real-valued function is increasing (isotone) if $a_{1} \preceq a_{2}$ implies $\omega\left(a_{1}\right) \leq \omega\left(a_{2}\right)$ and decreasing (antitone) if $a_{1} \preceq a_{2}$ implies $\omega\left(a_{1}\right) \geq \omega\left(a_{2}\right)$.

Theorem $3([\mathbf{1 3}, \mathbf{1 9}, \mathbf{1 2}])$. Let $\omega$ be a real-valued function on a concept lattice $(\mathbb{C}, \preceq)$. Then the function defined as:

$$
\forall\left(a_{1}, a_{2}\right) \in \mathbb{C}^{2}, d_{\omega}\left(a_{1}, a_{2}\right)=2 \omega\left(a_{1} \wedge a_{2}\right)-\omega\left(a_{1}\right)-\omega\left(a_{2}\right)
$$

is a pseudo-metric if and only if $\omega$ is a decreasing upper valuation.

The function defined as:

$$
\forall\left(a_{1}, a_{2}\right) \in \mathbb{C}^{2}, d_{\omega}\left(a_{1}, a_{2}\right)=\omega\left(a_{1}\right)+\omega\left(a_{2}\right)-2 \omega\left(a_{1} \vee a_{2}\right)
$$

is a pseudo-metric if and only if $\omega$ is a decreasing lower valuation. 
Owing to this result, one can obtain metrics by first defining suitable valuations on $(\mathbb{C}, \preceq)$. In what follows we introduce some examples of such valuations. We denote by $|\cdot|$ the cardinality (and restrict ourselves to the finite case).

Proposition 2. On $(\mathbb{C}, \preceq)$ associated with $\mathbb{K}=(G, M, I)$, the real-valued function defined as:

$$
\forall a \in \mathbb{C}, \omega_{G}(a)=|G|-|e(a)|
$$

is a strictly decreasing upper valuation.

Proof. Let $a_{1}=\left(X_{1}, Y_{1}\right)$ and $a_{2}=\left(X_{2}, Y_{2}\right)$ be two formal concepts. The antimonotonicity of $\omega_{G}$ follows from the fact that: $\left(X_{1}, Y_{1}\right) \preceq\left(X_{2}, Y_{2}\right)$ implies $X_{1} \subseteq X_{2}$, hence $|G|-\left|X_{1}\right| \geq|G|-\left|X_{2}\right|$. Let us now prove that $\omega_{G}$ is an upper valuation, i.e. it satisfies the submodular property.

From Equation 1 we have:

$$
\omega_{G}\left(a_{1} \wedge a_{2}\right)+\omega_{G}\left(a_{1} \vee a_{2}\right)=2|G|-\left|X_{1} \cap X_{2}\right|-\left|\beta\left(\alpha\left(X_{1} \cup X_{2}\right)\right)\right|
$$

then

$$
\begin{aligned}
& \omega_{G}\left(a_{1}\right)+\omega_{G}\left(a_{2}\right)-\omega_{G}\left(a_{1} \wedge a_{2}\right)-\omega_{G}\left(a_{1} \vee a_{2}\right) \\
= & \left|X_{1} \cap X_{2}\right|-\left|X_{1}\right|-\left|X_{2}\right|+\left|\beta\left(\alpha\left(X_{1} \cup X_{2}\right)\right)\right| \\
= & \left|\beta\left(\alpha\left(X_{1} \cup X_{2}\right)\right)\right|-\left|X_{1} \cup X_{2}\right| \\
\geq & 0
\end{aligned}
$$

since the closure operator $\beta \alpha$ is extensive $(X \subseteq \beta(\alpha(X)))$. This completes the proof.

Proposition 3. The function defined as:

$$
\forall\left(a_{1}, a_{2}\right) \in \mathbb{C}^{2}, \quad d_{\omega_{G}}\left(a_{1}, a_{2}\right)=2 \omega_{G}\left(a_{1} \wedge a_{2}\right)-\omega_{G}\left(a_{1}\right)-\omega_{G}\left(a_{2}\right)
$$

is a metric on $(\mathbb{C}, \preceq)$.

Proof. From Theorem 3 and Proposition $2, d_{\omega_{G}}$ is a pseudo-metric. Let $a_{1}=$ $\left(X_{1}, Y_{1}\right), a_{2}=\left(X_{2}, Y_{2}\right)$ be formal concepts in $\mathbb{C}$. Then $d_{\omega_{G}}\left(a_{1}, a_{2}\right)$ can be written as:

$$
d_{\omega_{G}}\left(a_{1}, a_{2}\right)=\left|X_{1}\right|+\left|X_{2}\right|-2\left|X_{1} \cap X_{2}\right|=\left|X_{1} \cup X_{2}\right|-\left|X_{1} \cap X_{2}\right|
$$

and is then a metric on $\mathbb{C}$ since $\left|X_{1} \cup X_{2}\right|-\left|X_{1} \cap X_{2}\right|=0$ implies $a_{1}=a_{2}$.

Proposition 4. On $(\mathbb{C}, \preceq)$ associated with $\mathbb{K}=(G, M, I)$, the real-valued function defined as:

$$
\forall a \in \mathbb{C}, \omega_{M}(a)=|i(a)|
$$

is a strictly decreasing lower valuation.

The proof is the same as for $d_{\omega_{G}}$ and is therefore omitted here. 
Proposition 5. The function defined as:

$$
\forall\left(a_{1}, a_{2}\right) \in \mathbb{C}^{2}, \quad d_{\omega_{M}}\left(a_{1}, a_{2}\right)=\omega_{M}\left(a_{1}\right)+\omega_{M}\left(a_{2}\right)-2 \omega_{M}\left(a_{1} \vee a_{2}\right)
$$

is a metric on $(\mathbb{C}, \preceq)$.

Proof. As for $d_{\omega_{G}}\left(a_{1}, a_{2}\right), d_{\omega_{M}}\left(a_{1}, a_{2}\right)$ is a pseudo-metric from Theorem 3 and Proposition 4, and it can be written as $\left|Y_{1}\right|+\left|Y_{2}\right|-2\left|Y_{1} \cap Y_{2}\right|=\left|Y_{1} \cup Y_{2}\right|-\left|Y_{1} \cap Y_{2}\right|$. It is then a metric on $\mathbb{C}$ since $\left|Y_{1} \cup Y_{2}\right|-\left|Y_{1} \cap Y_{2}\right|=0$ implies $a_{1}=a_{2}$.

Other possible valuations and inherited distances on $\mathbb{C}$ are listed below. Note that some of these valuations were introduced in [14], and the proofs are therefore omitted.

- $\omega_{I}(a)=\left|I_{a}\right|$, the cardinality of the ideal generated by an element $a$ of $\mathbb{C}$, is increasing and supermodular (lower valuation). Then one can define a pseudo-metric as:

$$
d_{\omega_{I}}\left(a_{1}, a_{2}\right)=\omega_{I}\left(a_{1}\right)+\omega_{I}\left(a_{2}\right)-2 \omega_{I}\left(a_{1} \wedge a_{2}\right)
$$

(this is derived from the fact that $-\omega_{I}$ is a decreasing upper valuation and from Theorem 3 applied on $-\omega_{I}$ ).

- $\omega_{F}(a)=\left|F_{a}\right|$, the cardinality of the filter generated by an element $a$ of $\mathbb{C}$, is decreasing and supermodular (lower valuation). Then one can define a pseudo-metric as:

$$
d_{\omega_{F}}\left(a_{1}, a_{2}\right)=\omega_{F}\left(a_{1}\right)+\omega_{F}\left(a_{2}\right)-2 \omega_{F}\left(a_{1} \vee a_{2}\right) .
$$

- By generalizing the previous valuations, one can define the following ones: consider a non-negative real-valued function $f$ on $\mathbb{C}$, then the function defined as:

$$
\omega_{f}(a)=\sum_{b \preceq a} f(b)
$$

is an increasing lower valuation, and

$$
\omega^{f}(a)=\sum_{a \preceq b} f(b)
$$

is a decreasing lower valuations.

- Based on notions from the theory of graded lattices [3], we can equip $\mathbb{C}$, since it is complete and finite, with a height function $\ell$, defined as the supremum of the lengths of all chains that join the smallest element of the lattice to the considered element. This function is strictly increasing and satisfies the following property: if $b$ covers $a$ (i.e. $a \subset b$ and $\nexists c$ such that $a \subset c \subset b$ ), then $\ell(b)=\ell(a)+1$. Hence this function endows the concept lattice with a graded lattice structure. In a general graded lattice, a pseudo-metric can be defined as

$$
d\left(a_{1}, a_{2}\right)=\ell\left(a_{1}\right)+\ell\left(a_{2}\right)-2 \ell\left(a_{1} \wedge a_{2}\right) .
$$

Note that if the lattice is the power set of some set, with the inclusion relation as partial ordering, then $\ell$ is equivalent to the cardinality and $d$ is a metric. 
Illustration on the Numbers Example. Let us illustrate some of the introduced distances on the example in Figure 1. Let $a_{1}=(\{4,9\},\{c, s\})$ and $a_{2}=(\{1,9\},\{o, s\})$, then:

$$
\begin{aligned}
& -d_{\omega_{G}}\left(a_{1}, a_{2}\right)=\left|e\left(a_{1}\right) \cup e\left(a_{2}\right)\right|-\left|e\left(a_{1}\right) \cap e\left(a_{2}\right)\right|=|\{4,9,1\}|-|\{9\}|=2, \\
& -d_{\omega_{M}}\left(a_{1}, a_{2}\right)=\left|i\left(a_{1}\right) \cup i\left(a_{2}\right)\right|-\left|i\left(a_{1}\right) \cap i\left(a_{2}\right)\right|=|\{c, s, o\}|-|\{c\}|=2, \\
& -d_{\omega_{I}}\left(a_{1}, a_{2}\right)=\left|I_{a_{1}}\right|+\left|I_{a_{2}}\right|-2\left|I_{a_{1} \wedge a_{2}}\right|=4+3-2 \times 2=3, \\
& -d_{\omega_{F}}\left(a_{1}, a_{2}\right)=\left|F_{a_{1}}\right|+\left|F_{a_{2}}\right|-2\left|F_{a_{1} \vee a_{2}}\right|=4+4-2 \times 2=4 .
\end{aligned}
$$

\subsection{Operators on $\mathcal{P}(G)$ from Structuring Elements on $\mathbb{C}$}

In order to define explicit operations on the concept lattice, we will make use of particular erosions and dilations, called morphological ones [17], which involve the notion of structuring element, i.e. a binary relation $b$ between elements of $G$. For $g \in G$, we denote by $b(g)$ the set of elements of $G$ in relation with $g$. For instance, $b$ can represent a neighborhood system in $G$ or a distance relation. For a distance $d$ between elements of $G$, structuring elements can be defined as balls of this distance. The particularity here relies in the choice of the distance. The distance in $G$ will be derived from a distance in $\mathbb{C}$ and a mapping from $G$ into $\mathbb{C}$. Balls of the distance and neighborhoods will be derived accordingly. In the sequel, we will rely on the mapping $p_{\mathbb{C}}$ introduced in Definition 2 , and the link between the neighborhood systems in both spaces for introducing the underlying dilation and erosion.

Definition 9. Let us denote by $d_{\mathbb{C}}$ any metric defined on $\mathbb{C}$. We define $d$ on $G$ as:

$$
\forall\left(g_{1}, g_{2}\right) \in G^{2}, d\left(g_{1}, g_{2}\right)=d_{\mathbb{C}}\left(p_{\mathbb{C}}\left(g_{1}\right), p_{\mathbb{C}}\left(g_{2}\right)\right)
$$

Proposition 6. $d$ is a pseudo-metric in $G$.

Proof. Since $d_{\mathbb{C}}$ is a metric, it follows directly that $d$ is positive, symmetrical, satisfies the triangular inequality, and $\forall g \in G, d(g, g)=0$. However $d$ is not separable. As a counter-example, let us consider the lattice in Figure 1, and $g_{1}=5, g_{2}=7$. We have $p_{\mathbb{C}}\left(g_{1}\right)=p_{\mathbb{C}}\left(g_{2}\right)=(\{3,5,7\},\{o, p\})$ and $d\left(g_{1}, g_{2}\right)=0$ (but $g_{1} \neq g_{2}$ ).

Examples of metrics $d_{\mathbb{C}}$ on $\mathbb{C}$ can be found in Section 4.1, and can be used here to derive pseudo-metrics on $G$ using Equation 13.

Definition 10. Let $d$ be a pseudo-metric on $G$ defined from $d_{\mathbb{C}}$ as in Equation 13. An elementary structuring element (neighborhood) of each element of $G$ is defined as:

$$
\begin{aligned}
\forall g \in G, b(g) & =\left\{g_{1} \in G \mid d\left(g, g_{1}\right) \leq 1\right\} \\
& =\left\{g_{1} \in G \mid d_{\mathbb{C}}\left(p_{\mathbb{C}}(g), p_{\mathbb{C}}\left(g_{1}\right)\right) \leq 1\right\}
\end{aligned}
$$

We now define dilations and erosions on the lattice $(\mathcal{P}(G), \subseteq)$. 
Definition 11. The morphological dilation of a subset $X$ of $G$ with respect to $b$ is expressed as:

$$
\delta_{b}(X)=\{g \in G \mid b(g) \cap X \neq \emptyset\} .
$$

The morphological erosion of $X$ is expressed as:

$$
\varepsilon_{b}(X)=\{g \in G \mid b(g) \subseteq X\} .
$$

These definitions provide results in $\mathcal{P}(G)$. They can be further restricted to get results in $e(\mathbb{C})$, where $e(\mathbb{C})$ denotes the image of $\mathbb{C}$ by the extent mapping, by applying $\beta \alpha$ on the resulting subset (i.e. $\beta\left(\alpha\left(\delta_{b}(X)\right)\right)$ and $\left.\beta\left(\alpha\left(\varepsilon_{b}(X)\right)\right)\right)$.

These definitions extend to neighborhoods, dilations and erosions of any size $n\left(n \in \mathbb{R}^{+}\right)$:

$$
\begin{aligned}
b^{n}(g) & =\left\{g_{1} \in G \mid d\left(g, g_{1}\right) \leq n\right\}, \\
\delta_{b^{n}}(X) & =\left\{g \in G \mid b^{n}(g) \cap X \neq \emptyset\right\}, \\
\varepsilon_{b^{n}}(X) & =\left\{g \in G \mid b^{n}(g) \subseteq X\right\} .
\end{aligned}
$$

These definitions enjoy all classical properties of mathematical morphology (since they correspond to a standard construction from a neighborhood relation). Moreover we have the following properties:

Proposition 7. The dilation $\delta_{b}$ (and any $\delta_{b^{n}}$ ) is extensive (i.e. $\forall X \subseteq G, X \subseteq$ $\delta_{b}(X)$ ). The erosion $\varepsilon_{b}$ (and any $\varepsilon_{b^{n}}$ ) is anti-extensive (i.e. $\forall X \subseteq G, \varepsilon_{b}(X) \subseteq X$ ).

Proof. This follows directly from the fact that $\forall g \in G, d(g, g)=0$ and hence $g \in b(g)$.

Note that for $n=0$, the dilation (respectively the erosion) does not reduce to the identity mapping. This comes from the fact that $d$ is only a pseudo-metric (i.e. non separable), and $\left\{g_{1} \in G \mid d\left(g, g_{1}\right)=0\right\}$ is not reduced to $g$.

Similar definitions on the lattice $(\mathcal{P}(M), \subseteq)$ can be provided. The construction and the properties are similar, by replacing $p_{\mathbb{C}}$ by $q_{\mathbb{C}}$ (cf. Definition 2$)$, and are therefore not detailed here.

Now from operations on $\mathcal{P}(G)$ and $\mathcal{P}(M)$, we can derive operators on $\mathbb{C}$. We suggest here a few ways to do so:

- Since $\mathbb{C}$ is sup-generated by the set $p_{\mathbb{C}}(G)$, it is sufficient to define a dilation $\delta_{p}$ on the image of $G$ by $p_{\mathbb{C}}$, and then use the sup-generating property and the definition of dilation as an operator that commutes with the supremum to derive:

$$
\forall a \in \mathbb{C}, \delta(a)=\bigvee_{g \in e(a)} \delta_{p}\left(p_{\mathbb{C}}(g)\right) .
$$

The definition of $\delta_{p}\left(p_{\mathbb{C}}(g)\right)$ can be built for instance from a valuation. Note however that the decomposition using $p_{\mathbb{C}}$ does not always lead to "simpler" elements ${ }^{6}$.

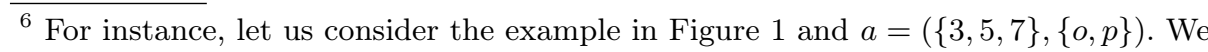
have $p_{\mathbb{C}}(3)=p_{\mathbb{C}}(5)=p_{\mathbb{C}}(7)=a$.
} 
- Similarly, since $\mathbb{C}$ is inf-generated by the $q_{\mathbb{C}}(m)$, an erosion $\varepsilon_{q}$ can be defined on the image of $M$ by $q_{\mathbb{C}}$ and then an erosion on $\mathbb{C}$ is defined as:

$$
\forall a \in \mathbb{C}, \varepsilon(a)=\bigwedge_{m \in i(a)} \varepsilon_{q}\left(q_{\mathbb{C}}(m)\right) .
$$

Note that if $\left(\varepsilon_{q}, \delta_{p}\right)$ forms an adjunction, then $(\varepsilon, \delta)$ is an adjunction.

- Another possibility is to build a dilation from the lattice $(\mathbb{C}, \preceq)$ to the lattice $(\mathcal{P}(G), \mathcal{P}(M), \preceq)$ from $\delta_{b}$ and $\varepsilon_{b}: \forall a \in \mathbb{C}, \delta(a)=\left(\delta_{b}(e(a)), \varepsilon_{b}(i(a))\right.$, and the adjoint erosion: $\forall a \in \mathbb{C}, \varepsilon(a)=\left(\varepsilon_{b}(e(a)), \delta_{b}(i(a))\right.$.

- Similarly dilations and erosions in the lattice $(\mathbb{C}, \preceq)$ can be defined by using additionally the derivation operators to ensure that the results are in $\mathbb{C}$, e.g. $\delta(a)=\left(\beta \alpha\left(\delta_{b}(e(a))\right), \alpha\left(\delta_{b}(e(a))\right)\right)=\left(\beta \alpha\left(\delta_{b}(e(a))\right), \alpha\left(\delta_{b}(\beta(i(a)))\right)\right)$.

We do not further detail these possibilities here. The next section details other examples of operators on $(\mathbb{C}, \preceq)$, based on different decompositions of formal concepts.

\subsection{Operators from Distances on $(\mathbb{C}, \preceq)$}

In this section we define morphological operators directly on $\mathbb{C}$. We introduce two approaches, both based on the property that $\mathbb{C}$ is sup-generated by the subset of join-irreducible elements.

\section{Join-Meet Generating Operators}

Let us discuss the dilation case. A first idea, exploiting the sup-generating property of $\mathbb{C}$, is to define an elementary dilation on join-irreducible elements of the lattice, and then derive dilation for any element in the lattice as a supremum of its join elements dilation. This is similar to the definition in Equation 18, except that the decomposition is different. We rely here as mentioned above on the join-irreducible decomposition of the element to dilate as stated in Definition 3.

A dilation on $\mathbb{C}$ is then based on two steps. We first define a distance-based elementary dilation of join-irreducible elements as:

$$
\forall a \in \mathcal{J}(\mathbb{C}), \delta^{\prime}(a)=\bigvee\{b \in \mathbb{C} \mid d(a, b) \leq n\}
$$

with $\mathcal{J}(\mathbb{C})$ as introduced in Definition 3, and then derive a dilation on $\mathbb{C}$ by using the sup-generating properties and the fact that a dilation commutes with the supremum.

In the following, to simplify notations, $\mathcal{J}(a)$ will denote a mapping of a concept $a \in \mathbb{C}$ to a minimal join-irreducible decomposition of $a(\mathcal{M}(a)$ is defined analogously).

Proposition 8. Let $\delta^{\prime}$ be an elementary dilation defined on $\mathcal{J}(\mathbb{C})$. Then the following mapping:

$$
\delta_{d}: \mid \begin{aligned}
& \mathbb{C} \longrightarrow \mathbb{C} \\
& a \longmapsto \bigvee_{b \in \mathcal{J}(a)} \delta^{\prime}(b)
\end{aligned}
$$


is a dilation.

Note that when $a$ is join-irreducible then $\delta_{d}(a)=\delta^{\prime}(a)$.

Let us now define the erosion operator by following the same pattern as for dilation. Let $\varepsilon^{\prime}$ be an elementary erosion defined on $\mathcal{M}(\mathbb{C})$ as:

$$
\forall a \in \mathcal{M}(\mathbb{C}), \varepsilon^{\prime}(a)=\bigwedge\{b \in \mathbb{C} \mid d(a, b) \leq n\}
$$

Proposition 9. Let $\varepsilon^{\prime}$ be an elementary erosion defined on $\mathcal{M}(\mathbb{C})$. Then the following mapping:

$$
\varepsilon_{d}: \mid \begin{aligned}
& \mathbb{C} \longrightarrow \mathbb{C} \\
& a \longmapsto \bigwedge_{b \in \mathcal{M}(a)} \varepsilon^{\prime}(b)
\end{aligned}
$$

is an erosion.

\section{Operators based on $\vee, \wedge$-Compatible Distances}

Based on the decomposition into irreducible elements, one can also define dilations and erosions using set-like distances ${ }^{7}$ that satisfy some compatibility criteria. In this section we discuss these compatibility criteria and provide some examples of set-like distances and their associated dilations and erosions.

Definition 12. A distance is said to be $\wedge$-compatible, and denoted by $d^{\wedge}$, if for any $n$ in $\mathbb{R}^{+}$and any family $\left(a_{i}\right)$ of elements of $\mathbb{C}$ :

$$
\left\{b \in \mathbb{C} \mid d^{\wedge}\left(\wedge_{i} a_{i}, b\right) \leq n\right\}=\bigcap_{i}\left\{b \in \mathbb{C} \mid d^{\wedge}\left(a_{i}, b\right) \leq n\right\}
$$

Definition 13. A distance is said to be $\vee$-compatible, and denoted by $d^{\vee}$, if for any $n$ in $\mathbb{R}^{+}$and any family $\left(a_{i}\right)$ of elements of $\mathbb{C}$ :

$$
\left\{b \in \mathbb{C} \mid d^{\vee}\left(\vee_{i} a_{i}, b\right) \leq n\right\}=\bigcup_{i}\left\{b \in \mathbb{C} \mid d^{\vee}\left(a_{i}, b\right) \leq n\right\} .
$$

Proposition 10. Let $d$ be any distance on the concept lattice $(\mathbb{C}, \preceq)$, and $\mathcal{J}$ the join-irreducible decomposition operator on $(\mathbb{C}, \preceq)$, then the following operator:

$$
\forall(a, b) \in \mathbb{C}^{2}, d^{\vee}(a, b)=\inf _{a_{i} \in \mathcal{J}(a)} d\left(a_{i}, b\right)
$$

is $\vee$-compatible.

Proof. We have: $\left\{b \mid d^{\vee}(a, b) \leq n\right\}=\left\{b \mid \inf _{a_{i} \in \mathcal{J}(a)} d\left(a_{i}, b\right) \leq n\right\}=\left\{b \mid \exists a_{i} \in\right.$ $\left.\mathcal{J}(a), d\left(a_{i}, b\right) \leq n\right\}=\bigcup_{i}\left\{b \mid d\left(a_{i}, b\right) \leq n\right\}=\bigcup_{i}\left\{b \mid d^{\vee}\left(a_{i}, b\right) \leq n\right\}$, since for any join-irreducible element $a_{i}, d^{\vee}\left(a_{i}, b\right)=d\left(a_{i}, b\right)$. The result generalizes to any supremum $\vee_{i} a_{i}$ of (possibly reducible) elements, by virtue of associative and commutative properties of the $\bigcup$ operator.

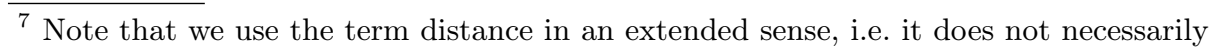
satisfy all metrics properties.
} 
Proposition 11. Let $d$ be any distance on the concept lattice $(\mathbb{C}, \preceq)$, and $\mathcal{J}$ the join-irreducible decomposition operator on $(\mathbb{C}, \preceq)$, then the following operator:

$$
\forall(a, b) \in \mathbb{C}^{2}, d^{\wedge}(a, b)=\sup _{a_{i} \in \mathcal{J}(a)} d\left(a_{i}, b\right)
$$

is $\wedge$-compatible.

Proof. We have: $\left\{b \mid d^{\wedge}(a, b) \leq n\right\}=\left\{b \mid \sup _{a_{i} \in \mathcal{J}(a)} d\left(a_{i}, b\right) \leq n\right\}=\left\{b \mid \forall a_{i} \in\right.$ $\left.\mathcal{J}(a), d\left(a_{i}, b\right) \leq n\right\}=\bigcap_{i}\left\{b \mid d\left(a_{i}, b\right) \leq n\right\}=\bigcap_{i}\left\{b \mid d^{\wedge}\left(a_{i}, b\right) \leq n\right\}$, since for any join-irreducible element $a_{i}, d^{\wedge}\left(a_{i}, b\right)=d\left(a_{i}, b\right)$. The result generalizes to any supremum $\vee_{i} a_{i}$ of (possibly reducible) elements, by virtue of associative and commutative properties of the $\bigcap$ operator.

Furthermore $d^{\wedge}$ and $d^{\vee}$ satisfy the identity property, i.e. $d^{\wedge}(a, a)=0$ and $d^{\vee}(a, a)=0$, if $d$ does, but they are not symmetrical.

Proposition 12. Let $\mathbb{C}$ be a complete concept lattice, and let $d^{\vee}$ be $a \vee$-compatible distance on $(\mathbb{C}, \preceq)$. For any $n$ in $\mathbb{N}$, the operator expressed as:

$$
\delta_{c}: \mid \begin{aligned}
& \mathbb{C} \longrightarrow \mathbb{C} \\
& a \longmapsto \bigvee\left\{b \in \mathbb{C} \mid d^{\vee}(a, b) \leq n\right\}
\end{aligned}
$$

is a dilation.

Proof. This follows directly from the $\vee$-compatibility of $d^{\vee}$ and from the following property: for all $A, B \subseteq \mathbb{C}, \bigvee(A \cup B)=(\bigvee A) \vee(\bigvee B)$

Proposition 13. Let $\mathbb{C}$ be a complete concept lattice, and let $d^{\wedge}$ be a $\wedge$-compatible distance on $\mathbb{C}$. For any $n$ in $\mathbb{N}$, the operator expressed as:

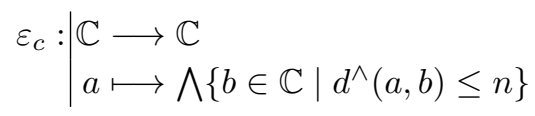

is an erosion.

Proof. This follows directly from the $\wedge$-compatibility of $d^{\wedge}$ and from the following property: for all $A, B \subseteq \mathbb{C}, \wedge(A \cap B)=(\bigwedge A) \wedge(\bigwedge B)$.

\section{Conclusion}

In this paper, we proposed to establish links between FCA theory and mathematical morphology. Based on preliminary observations of similar properties in both domains, we defined several morphological operators on the power set of objects (or attributes) on the one hand, and on the concept lattice itself on the other hand. These definitions are based on valuations, neighborhood relations, and distances. The proposed operators have good properties in terms of mathematical morphology, which can now be exploited for developing reasoning services on the concept lattice. Other possible approaches will also be developed in our future work. For instance, mathematical morphology on graphs or hypergraphs can be used on the graph representing the lattice, or on a bipartite graph (from which a hypergraph can be derived) built from objects and attributes. 


\section{References}

1. Atif, J., Hudelot, C., Bloch, I.: Abduction in description logics using formal concept analysis and mathematical morphology: application to image interpretation. In: 8th International Conference on Concept Lattices and Their Applications (CLA2011). pp. 405-408. Nancy, Paris (Oct 2011)

2. Baader, F.: Computing a minimal representation of the subsumption lattice of all conjunctions of concepts defined in a terminology. In: Knowledge Retrieval, Use and Storage for Efficiency: 1st International KRUSE Symposium. pp. 168-178 (1995)

3. Birkhoff, G.: Lattice theory (3rd edition), vol. 25. American Mathematical Society (1979)

4. Bloch, I.: On Links between Mathematical Morphology and Rough Sets. Pattern Recognition 33(9), 1487-1496 (2000)

5. Bloch, I., Heijmans, H., Ronse, C.: Mathematical Morphology. In: Aiello, M., PrattHartman, I., van Benthem, J. (eds.) Handbook of Spatial Logics, chap. 13, pp. 857-947. Springer (2007)

6. Bloch, I., Maître, H.: Fuzzy Mathematical Morphologies: A Comparative Study. Pattern Recognition 28(9), 1341-1387 (1995)

7. Bloch, I., Pino-Pérez, R., Uzcategui, C.: A Unified Treatment of Knowledge Dynamics. In: International Conference on the Principles of Knowledge Representation and Reasoning, KR2004. pp. 329-337. Canada (2004)

8. Ferré, S., Ridoux, O.: A logical generalization of formal concept analysis. Conceptual Structures: Logical, Linguistic, and Computational Issues pp. 371-384 (2000)

9. Ganter, B., Wille, R., Franzke, C.: Formal concept analysis: mathematical foundations. Springer-Verlag New York, Inc. (1997)

10. Heijmans, H.J.A.M.: Morphological Image Operators. Academic Press, Boston (1994)

11. Heijmans, H.J.A.M., Ronse, C.: The Algebraic Basis of Mathematical Morphology Part I: Dilations and Erosions. Computer Vision, Graphics and Image Processing 50, 245-295 (1990)

12. Leclerc, B.: Lattice valuations, medians and majorities. Discrete Mathematics 111(1), 345-356 (1993)

13. Monjardet, B.: Metrics on partially ordered sets-a survey. Discrete mathematics 35(1), 173-184 (1981)

14. Orum, C., Joslyn, C.: Valuations and metrics on partially ordered sets. arXiv preprint arXiv:0903.2679 (2009)

15. Ronse, C., Heijmans, H.J.A.M.: The Algebraic Basis of Mathematical Morphology Part II: Openings and Closings. Computer Vision, Graphics and Image Processing 54, 74-97 (1991)

16. Ronse, C.: Adjunctions on the lattices of partitions and of partial partitions Applicable Algebra in Engineering, Communication and Computing 21(5), 343-396 (2010)

17. Serra, J.: Image Analysis and Mathematical Morphology. Academic Press, New-York (1982)

18. Serra (Ed.), J.: Image Analysis and Mathematical Morphology, Part II: Theoretical Advances. Academic Press, London (1988)

19. Simovici, D.: Betweenness, metrics and entropies in lattices. In: 38th IEEE International Symposium on Multiple Valued Logic. ISMVL. pp. 26-31 (2008)

20. Stern, M.: Semimodular lattices: theory and applications. Cambridge University Press (1999) 